\title{
The Use of Ultrasound in the Placement of Totally Implantable Veinous Access Ports (TIVAP) for Chemotherapy
}

\section{EL Bouazzaoui Abderrahim ${ }^{1 *}$, Jabrane Marouane ${ }^{1}$, Touzani Soumaya ${ }^{1}$, Houari Nawfal ${ }^{1}$, Maaroufi Mustapha ${ }^{2}$, Tsigbe Wisdom Kwasi ${ }^{1}$, Boukatta Brahim $^{1}$ and Kanjaa Nabil ${ }^{1}$}

${ }^{1}$ Anesthesiology and Intensive Care Department A4, Hassan II University Hospital, Faculty of Medicine and Pharmacy, Sidi Mohammed Ben Abdellah University, Fez, Morocco

${ }^{2}$ Radiology Department, Hassan II, University Hospital. Faculty of Medicine and Pharmacy, Sidi Mohammed Ben Abdellah University, Fez, Morocco

*Corresponding Author: EL Bouazzaoui Abderrahim, Anesthesiology and Intensive Care Department A4, Hassan II University Hospital, Faculty of Medicine and

Pharmacy, Sidi Mohammed Ben Abdellah University, Fez, Morocco.
Received: December 02, 2021

Published: December 15, 2021

(C) All rights are reserved by EL Bouazzaoui Abderrahim., et al.

\begin{abstract}
Introduction: There is an increasing demand for Totally Implantable Intravenous Access Ports (TIVAP), mainly to facilitate the initiation of intravenous chemotherapy. Ultrasound guidance during venipunctures for the placement of these TIVAP catheters is a good technique that is highly recommendable for Practicians. We present a prospective study for the placement of TIVAP using Ultrasound-guided percutaneous approach in 288 patients. The objective of this study is to demonstrate the contribution of Ultrasound guidance in making the procedure for the placement of these devices a safe one and also to study the complications that may arise from the placement of these intravenous devices.
\end{abstract}

Patients and Methods: This is a prospective study over a period of 7 years from January 2015 until July 2021 carried out in the A4 multipurpose anesthesia-intensive care unit of the CHU Hassan II in Fez. All the TIVAP were inserted under Ultrasound guidance. The parameters studied were: the sex of the patient, age, the cancer to be treated, the puncture site, the side of the puncture, the ultrasound technique, the per- and post-operative complications that occurred during or after the placement of the intravenous device.

Results: The study includes 288 Totally Implantable Venous Access Ports (TIVAP) placed in 288 patients of which 52.7\% male and $47.2 \%$ female. The reasons for chemotherapy were diverse. Ultrasound exploration of the veins was systematic in all patients before starting the implantation procedure. Two cases of venous thrombosis were incidentally diagnosed during these preoperative explorations. Venipuncture was performed at the axillary area in $67.70 \%$ of cases, against $31.5 \%$ of cases at the internal jugular area, and in the end $1.05 \%$ of cases of these punctures were at the femoral area. The ultrasound technique for venipuncture was in the ultrasound plane in $71.18 \%$ of cases, compared with $28.81 \%$ of venipunctures performed outside the ultrasound plane. Venipuncture was obtained during the first trial in $100 \%$ of cases. We did not observe any case of pneumothorax, arterial puncture or hematoma. The duration of the TIVAP procedure varied between 45 and 90 minutes depending on the case. Four cases of aberrant path of the TIVAP catheter were observed on the chest X-rays; which was systematically taken at the end of the insertion procedures. These aberrant paths were subsequently corrected via endovascular route by interventional radiology. In the long term, we noted one case of extravasation of the chemotherapy product, two cases of surgical site infection and one case of catheter exposure.

Discussion and Conclusion: The use of ultrasound decreases the risk of pleural damage and the formation of pneumothorax. Ultrasound guidance also decreases the risk of hematoma by reducing the number of punctures and avoiding arterial punctures. Ultrasound exploration also helps to verify the anatomical location of the vein, its permeability and the presence or absence of deep vein thrombosis. Thus, the use of ultrasound makes the procedure for inserting TIVAP a safe and secured one.

Keywords: Totally Implantable Venous Access Ports (TIVAP); X-ray; Ultrasound; Chemotherapy 


\section{Introduction}

Totally Implantable Venous Access Ports (TIVAP) emerged about fifteen years ago, they were used as a device to take care of patients requiring the administration of intensive or prolonged Chemotherapy treatments, parenteral nutrition, antibiotherapy or the transfusion of blood and its derivatives. There are numerous ways of inserting a TIVAP, multiple venous access routes can be used; the internal jugular vein route, the subclavian vein route or from a peripheral vein such as the external jugular vein. The use of Ultrasound guidance for TIVAP placement is a recommendable technique for Practicians; it is in this light that we proceeded to carry out a retrospective study on the placement of TIVAP using Ultrasound guided percutaneous approach in 288 Patients. The aim of this article is to describe the placement of TIVAP using Ultrasound guidance, to evaluate its efficacy and morbidity and then make a case through literature review on the importance of Ultrasound and finally make recommendations that may help prevent complications linked to the procedure.

\section{Material and Methods}

This is a prospective study over a period of 7 years starting from January 2015 until July 2021 carried out in the Multipurpose Anesthesia-intensive care Service A4 of the Hassan II University Hospital in Fes. The performance of the procedure was scheduled on an out-patient basis. The patients were transferred to our various training facilities and for each procedure we established a form on which all information were noted. Each patient was informed about the procedure and the placement technique. The parameters studied were; sex, age, the type of Cancer to treat, the puncture site, the side of the puncture, the Ultrasound guidance technique, and the perioperative and post-operative complications which occurred during the duration of the study.

\section{Results}

During the period of study 288 patients had Totally Implantable Veinous Access Ports (TIVAP) inserted in them using Ultrasound guidance, of which 152 patients were Males representing $52.7 \%$ of total cases and 136 patients were Females representing 47.2\% of total cases with a Male to Female sex ratio of 1.1. The mean age of our patients was 61.8 years ranging from 28 to 90 years. In this study, the predominant diagnosis was Breast Cancer in 82 Patients representing $28.4 \%$ of total cases, followed by Non-hodgkin Lymphoma in 76 Patients representing $26.3 \%$ of total cases, then gastric and rectal adenocarcinoma in $12.5 \%$ of total cases or in 35 patients. 24 cases, that is $8.3 \%$ of total cases were carriers of epidermoid car- cinoma of the Larynx and 13 cases, representing $4.51 \%$ were carriers of Colic Adenocarcinoma. For the rest of the Patients, we had 15 cases of Pancreatic Adenocarcinoma (5.2\%), 5 Cases(1.73\%) of Epidermoid Carcinoma of the Buccal Cavity and Epidermoid Carcinoma of the Nasopharynx. Lastly, 3 cases, that is $1.04 \%$ of total cases were Mediastinal Lymphoma Complicated by Superior Vena Cava Syndrome. Each Patient had lidocaine2\% administered on them, which is a local anesthetic agent before the start of the Procedure. The needle puncture was performed on the internal jugular vein (Figure 1) in 90 patients or $31.25 \%$ and on the axillary vein (Figure 2) in 195 Patients (67.70\%) and in 3 Patients who had mediastinal Lymphoma complicated by Superior Vena Cava Syndrome, the insertion site was femoral. In $94.79 \%$ of cases, the insertion took place on the right side as against $5.20 \%$ on the left side. The venipuncture whether jugular or axillary, on the right as on the left, was obtained on the first attempt in $100 \%$ of cases, without iterative puncture. Ultrasound guidance was used systematically in all our patients. The patient was placed in a supine position with both arms along the body, turning the head in opposite direction to the chosen side. The operator was positioned on the side of the chosen side of the puncture area. For ergonomy purposes, the ultrasound machine was placed in front of the operator (Figure 3). For the internal jugular vein access, the Ultrasound probe was placed on the sternocleidomastoid muscle at $3 \mathrm{~cm}$ above the Clavicle, the puncture being done posteriorly behind the Clavicular head of the sternocleidomastoid muscle. For the axillary vein route a $2 \mathrm{~cm}$ incision was made at the deltopectoral groove and the ultrasound probe was placed beneath the Clavicle and the axillary vein puncture being done through cutaneous incision either in the plane or outside the plane of the ultrasound (Figures 4 and 5). The location of the different anatomical structures is carried out from the beginning to determine the position of the artery in relation to the vein. The latter is spotted in the form of a hypoechogenic circular image, depressible under the pressure of the ultrasound probe. Possible Lymphadenopathy are also identified. After local anesthesia with lidocaine, the puncture needle, mounted on a syringe containing physiological saline solution, was then introduced under ultrasound guidance, in or out of the ultrasound probe plane. We then visualise the hyperechogenic needle which progresses into the vein. As soon as the end of the needle is in the vein, a reflux of venous blood appears. The syringe is then withdrawn and the metallic guide wire is introduced. After having withdrawn the needle, we introduce the needle thread on which we mount the dilator (Figure 7). We then remove the needle thread and insert the catheter. The port was then inserted in a cavity with two crossed-fingers beneath the clavicle (Figure 8,9). A permeability check of the system is essential(Blood Suction), then rinsing 
of the catheter with physiological saline solution was carried out. The subclavicular incision was closed in two planes, with an absorbable suture subcutaneously and an intradermal suture on the skin. 3 cases of Deep Vein Thrombosis was identified during Ultrasound exploration of the internal jugular vein, for which the TIVAP procedure was postponed until after an anticoagulant therapy (Figure 10). The majority of our patients, $71.18 \%$ of cases, had needle puncture in the ultrasound plane, compared to $28.81 \%$ of patients outside the ultrasound plane. The duration of the placement of the TIVAP varied between 45 and 90 minutes depending on the case. We did not observe any cases of pneumothorax or arterial puncture or hematoma. We observed two cases of guidewire passage from the punctured axillary vein to the ipsilateral internal jugular vein (Figure 11). As a result of this ultrasound finding, the guidewire was then redirected into the upper vena cava system. After the insertion procedure, a chest X-ray was systematically requested to verify the correct positioning of the catheter (Figure 12). Thus, four cases of aberrant path of the TIVAP catheter were diagnosed on the chest $\mathrm{X}$-ray, one of which was towards the ipsilateral jugular vein and three cases towards the contralateral brachiocephalic vein (Figure 13). A case of TIVAP catheter kink was observed (Figure 14). These patients were programmed for interventional radiology for repositioning of the catheter by endovascular route through percutaneous approach of the femoral vein (Figure 15). After a period of time; we identified a case of extravasation of the chemotherapy product at a distance from the insertion site (Figure 16), two cases of infection at the incision area (Figure 17) and an exposure of the catheter in one case (Figure 18).

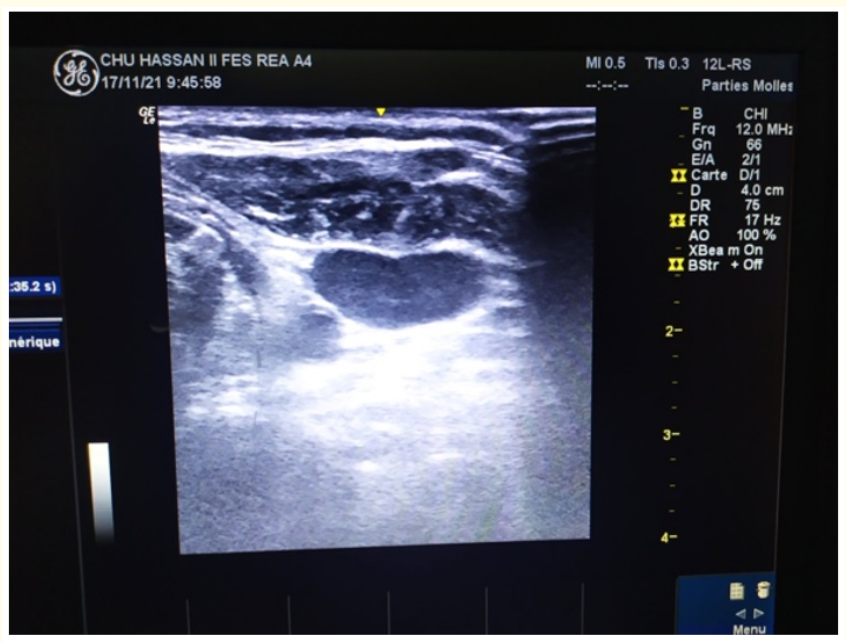

Figure 1: Ultrasound visualization of the right internal jugular vein.
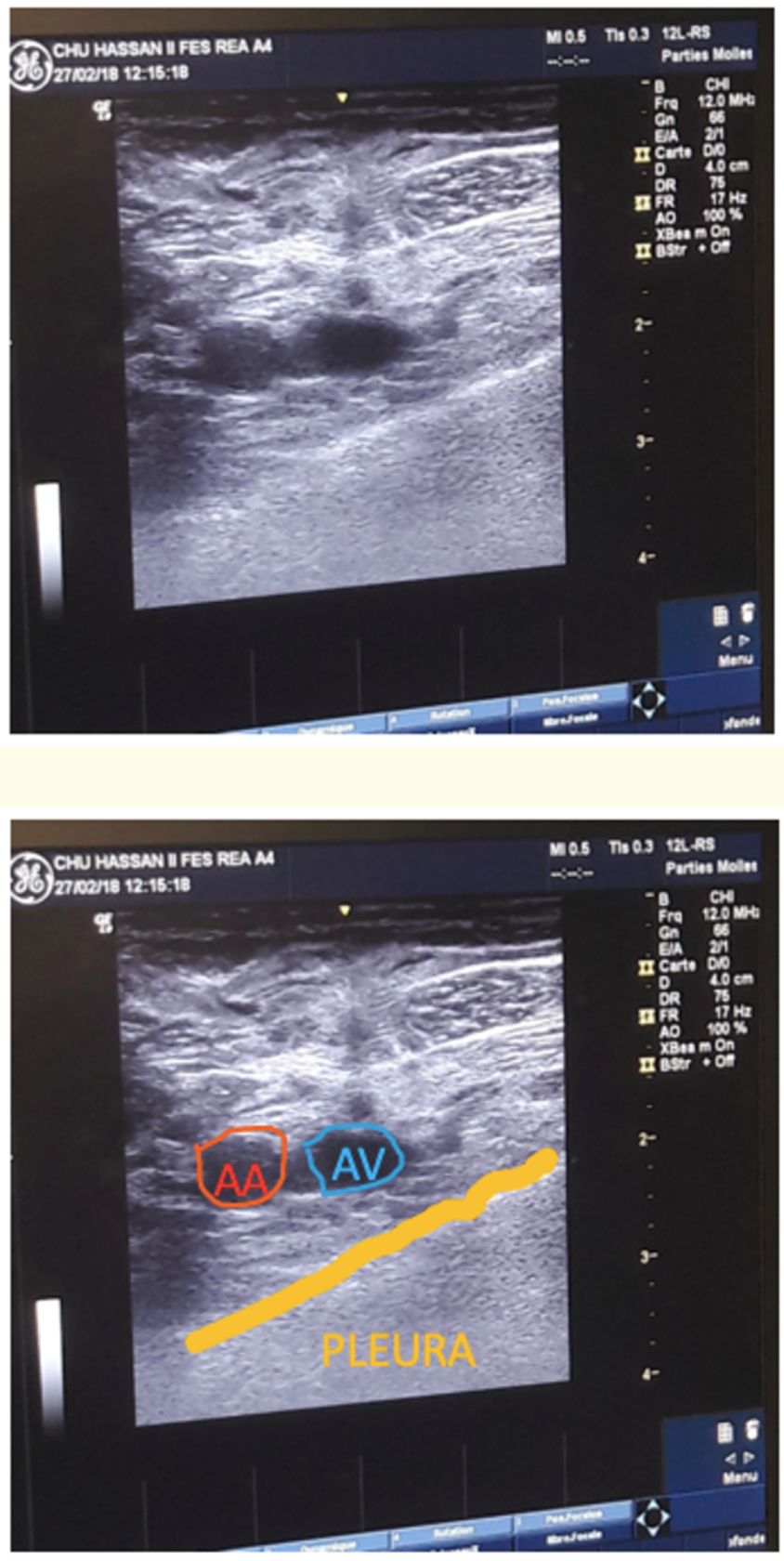

Figure 2: Out-of-plane visualization of the ultrasound of the right axillary vein (AV in blue). Notice the close relationship with the axillary artery (AA in red) and the pleura (yellow line). 

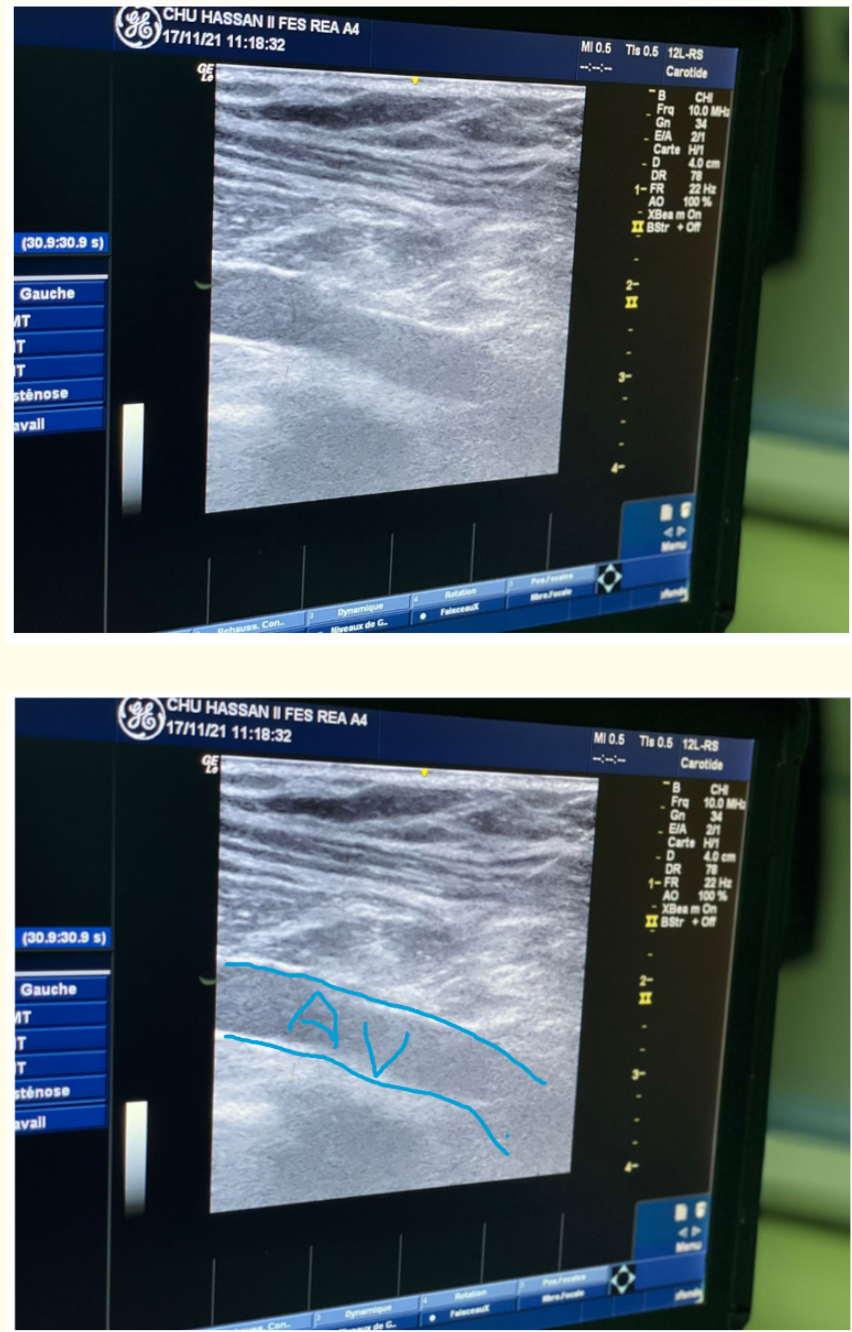

Figure 3: Visualization in the ultrasound plane of the right axillary vein (AV in blue).

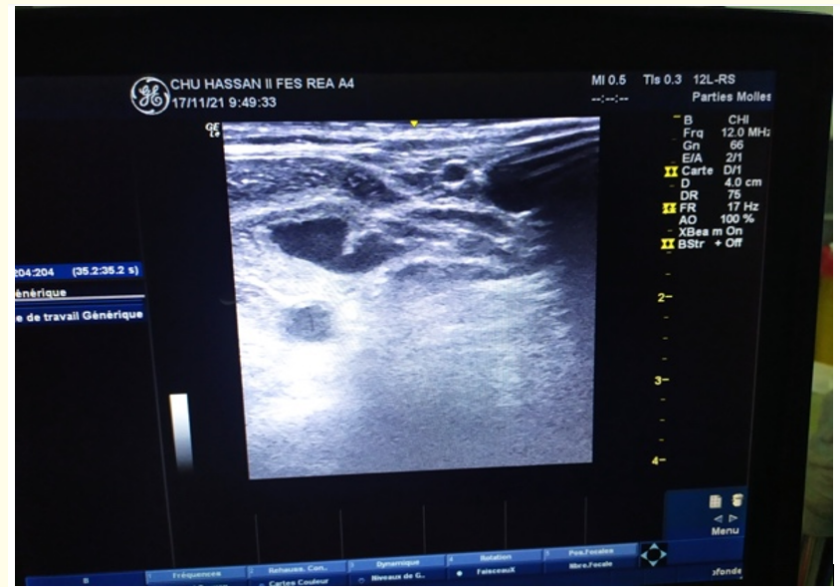

Figure 4: Ultrasound-guided puncture of the right internal jugular vein. Notice the out-of-plane arrival of ultrasound from the puncture needle into the jugular vein. The puncture needle is in the center of the lumen of the vein.
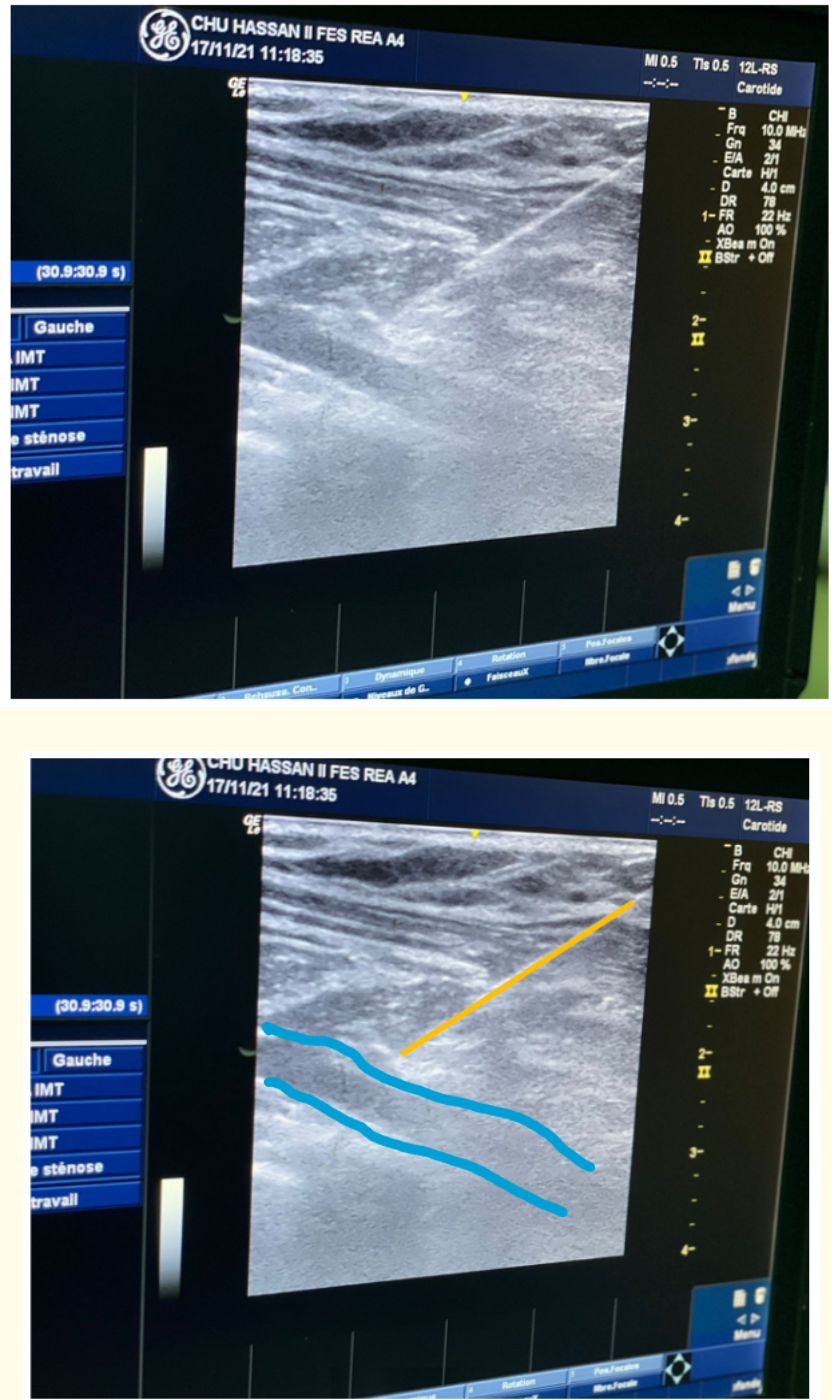

Figure 5: Ultrasound-guided puncture of the right axillary vein. Notice the arrival in the ultrasound plane of the puncture needle (in yellow) and its progression towards the lumen of the axillary vein (in blue).

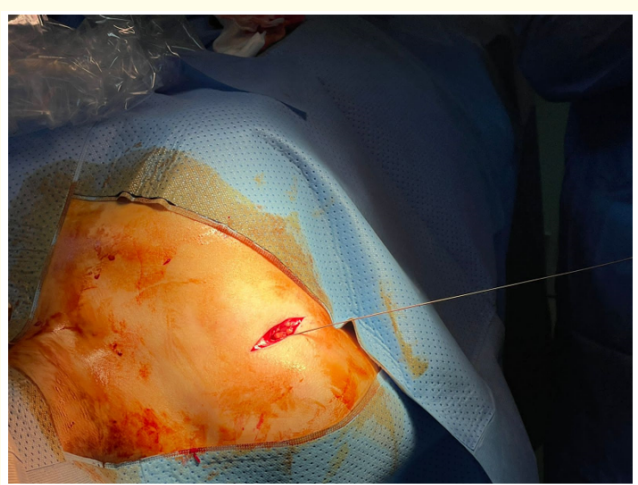

Figure 6: $4 \mathrm{~cm}$ incision in the right deltopectoral groove.

Puncture of the axillary vein through the delto pectoral incision, then introduction of the guide wire into the punctured vein. 


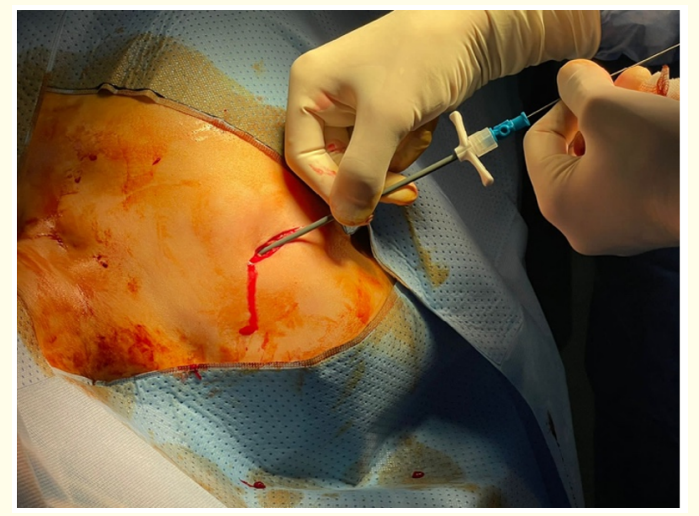

Figure 7: Introduction into the axillary vein of the dilator after mounting on the guidewire.

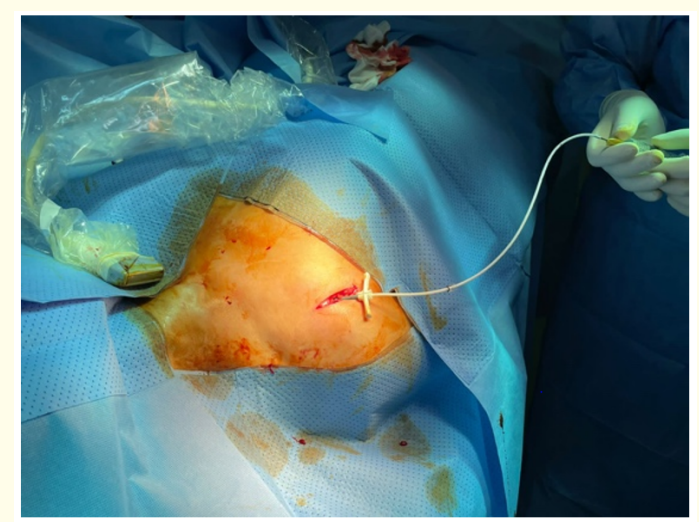

Figure 8: Introduction of the catheter into the axillary vein through the dilator.

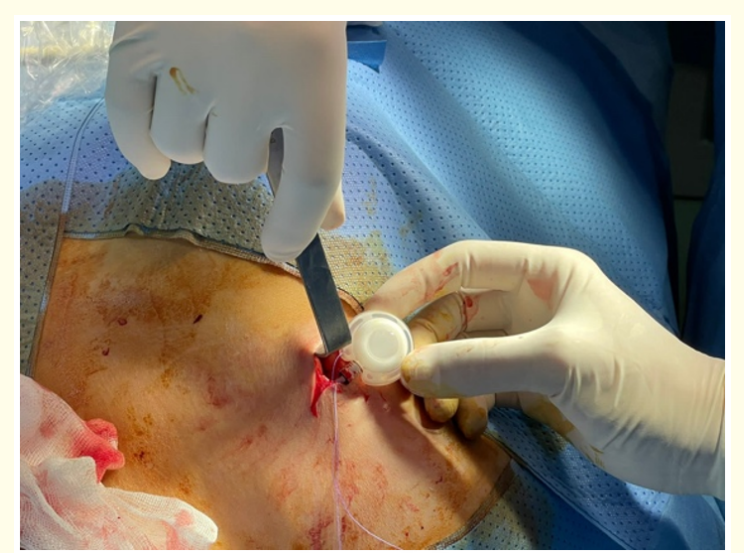

Figure 9: Cut the catheter to the correct size, connect the port to the catheter and insert the device subcutaneously.

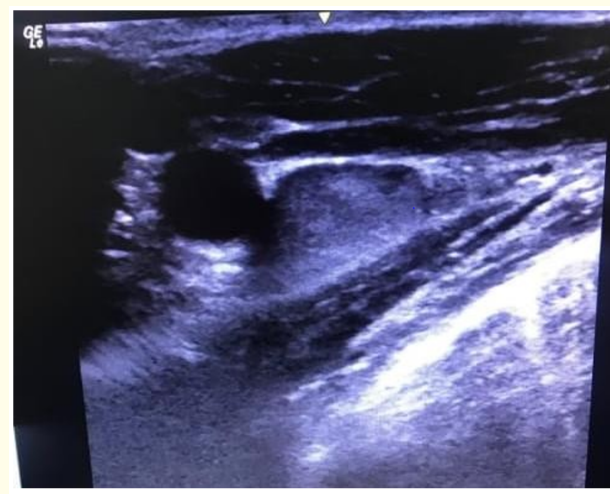

Figure 10: Pre-procedural exploration of the venous network. Fortuitous discovery of subclinical axillary vein thrombosis.

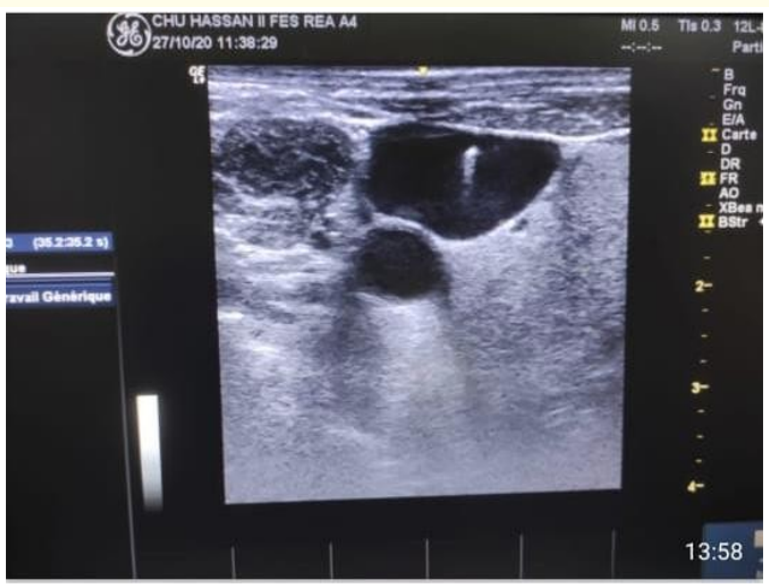

Figure 11: Out-of-plane ultrasound view showing an aberrant path with passage of the guidewire from the punctured axillary vein to the ipsilateral internal jugular vein.

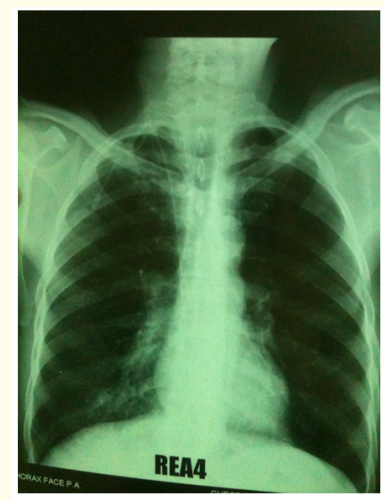

Figure 12: AP chest $x$-ray showing the positioning of the TIVAP catheter. 


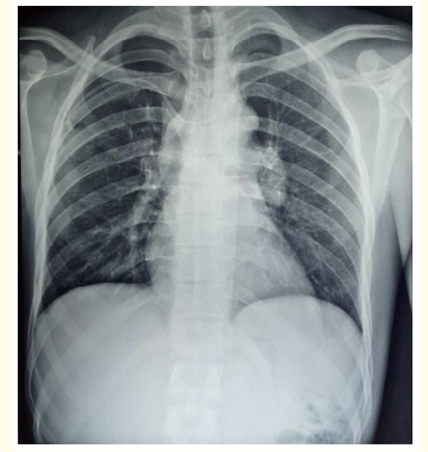

Figure 13: Frontal chest X-ray showing an aberrant path of a catheter placed through the right axillary venous route towards the contralateral brachiocephalic vein.

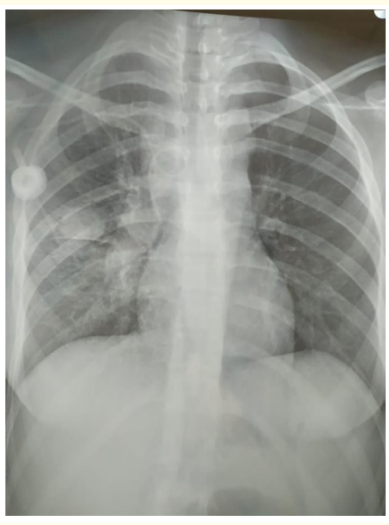

Figure 14: AP chest $x$-ray showing a kink of the catheter with an implantable chamber in the mediastinum.

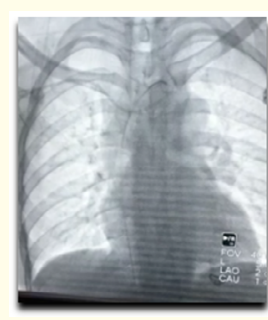

a

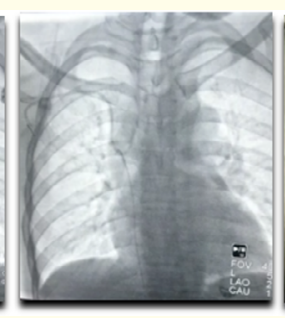

b

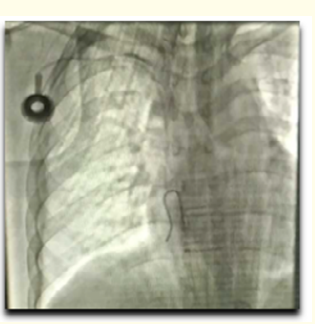

C
Figure 15: Endovascular procedure for correcting the position of a TIVAP catheter.

a: Puncture under echo guidance of the femoral vein and guide wire introduction into the superior vena cava system then into the left brachiocephalic vein of a catheter having one end preformed in a "U".

b: Hooking between the U-tip catheter and the TIVAP catheter in order to straighten the path of the catheter.

c: TIVAP catheter repositioned in the superior vena cava and removal of the U-tip catheter at the end of the procedure.

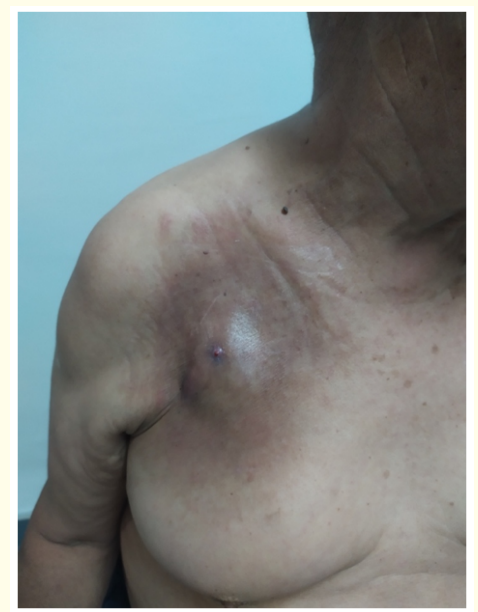

Figure 16: Illustration showing a case of extravasation of a chemotherapy product at a distance from the insertion site. Notice the toxic effect on the tissues surrounding the extravasation site.

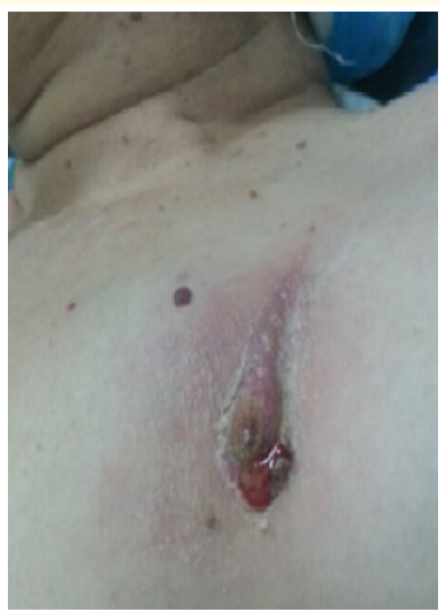

Figure 17: Infection of the insertion site of the TIVAP 3 months after placement.

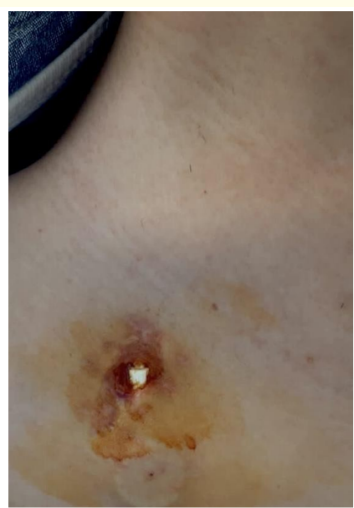

Figure 18: Exposure of a TIVAP catheter. 


\section{Discussion}

By allowing continuous visualization and identification of anatomical structures and pre-existing subclinical venous thrombosis, Ultrasound guidance increases the success rate of venipuncture irrespective of the venous access route, the success rate for the first venipuncture attempt using Ultrasound guidance is $96 \%$. The Ultrasound guidance also reduces the duration of the procedure as well as decrease the rate of initial Catheter misplacement [1,2]. In fact, the use of Ultrasound guidance decreases the risk of hematoma by reducing the number of punctures and avoiding arterial punctures. The use of Ultrasound guidance also decreases the risk of pleural lesion and the occurrence of Pneumothorax. In fact, in our study no case was complicated by pneumothorax or arterial puncture or hematoma due to the use of ultrasound guidance as against $1.48 \%$, $11.1 \%, 1.58 \%$ respectively in a series of 1008 TIVAP performed without ultrasound guidance reported by Hammoumi., et al. in 2014 [3]. Lefrant., et al. reported in a prospective series of 707 central venous catheters placed by surgical method, an incidence of pneumothorax of 3.1\% [4]. T Lmakinsi., et al. also reported in a prospective series of 101 catheters an incidence of pneumothorax of 3\% [5]. In our work, the incidence of pneumothorax was $0 \%$, thus underlining the primary importance of ultrasound guidance. The unprecedented contribution of ultrasound is also recently reported by Liang $\mathrm{Xu}$ and all in a study including sixty-seven breast cancer patients who underwent ultrasound-guided implantation of totally implantable venous access ports via the right innominate vein for administration of chemotherapy. In this study published in 2019, the success rate of the first attempt of venous puncture was $95.52 \%$ and no pneumothorax or hemothorax were observed [6]. The use of ultrasound also makes it possible to explore the venous network before starting the procedure for placing the device intravenously. Thus, it makes it possible to verify the anatomical location of the vein, its permeability and the presence or absence of subclinical venous thrombosis. The latter can be frequent in this population having risk factors for thrombosis such as cancerous disease, or ongoing chemotherapy. In our series, three cases of subclinical thrombosis in the internal jugular or axillary vein were accidentally diagnosed due to systematic ultrasound exploration of the venous network before starting the procedure for placing the TIVAP. This made it possible to avoid the puncture of these thrombosed veins, thus avoiding the occurrence of a serious adverse event secondary to the puncture of these thrombosed veins. Thus, the use of ultrasound is essential to make the procedure safe for the insertion of the TIVAP. An aberrant path is a possible complication of the catheter. In fact, when the catheter is placed in the axillary site, the latter is pushed not towards the end of the superior vena cava but it goes up to the jugular site. When the catheter is placed axillary, the aberrant path can be in the ipsilateral jugular vein or in the brachiocephalic vein against the lateral. In our series, aberrant pathways were observed in four patients who had hemopathy with mediastinal lymphadenopathy, that is $1.3 \%$ of cases versus $6.7 \%$ of cases according to Rouzrokh's study [7]. Only one case of catheter kinking was noted in our series. In the long term, the infection of the TIVAP remains the most frequent complication after thrombosis. Infection is the cause of a high percentage of withdrawals (20 to 50\% depending on the study), especially in cases of infection with Candida or Staphylococcus aureus. According to studies, the incidence of infections varies from 0.026 to 0.86 per 1000 catheter-days for all types of infections combined; from 0.016 to 0.24 per 1000 catheter days for bacteremia and 0.19 per 1000 catheter days for local infections. It should be noted, however, that the incidence is not always expressed in catheter days, the type of infection is not always specified and that, even though the most frequently described infection is the occurrence of bacteremia, the definitions adopted often vary from one study to another [810]. Ultrasound guidance reduces the risk of sepsis by reducing the number of puncture attempts and reducing the duration of the procedure. In fact, Se Jin Ahn and all retrospectively reviewed 1254 central venous ports implanted between August 2002 and October 2009. All procedures were guided by using ultrasound and fluoroscopy. Catheterization was performed via the right IJV in $95.5 \%$ of patients, and the left IJV in $4.5 \%$ of patients. A total of 8 infectious complications (0.64\%, 0.018/1000 catheter days) occurred. Four infections were early complications, and four were late complications as follows: catheter-related sepsis $(n=1)$, febrile catheterrelated bacteremia $(n=6)$, and port pocket infection $(n=1)$. Ports were immediately explanted in all infectious complications, except for one patient of transient febrile bacteremia which was treated with systemic antibiotics [11]. In our study, two cases of infection through a TIVAP catheter were reported, or $0.69 \%$ of cases. The infection was restricted to the puncture site without bacteremia or systemic infection. The extravasation or accidental leakage of cytotoxic product, from the compartment of the TIVAP or the venous system into the surrounding tissue which can be the most severe form of complication for the patient was observed in our study. It should be noted that the frequency of occurrence of extravasation during chemotherapy is 0.5 to $6 \%$. In our study, only one case of extravasation was reported, i.e. $0.34 \%$.

\section{Conclusion}

The use of Totally Implantable Venous Access Ports (TIVAP) is on the rise, hence, it is imperative to make the procedure safe, efficacious, reproducible, non-iatrogenic and minimally invasive as far 
as possible. The use of Ultrasound guidance enables us to respond to these imperatives so as to obtain maximum efficacy and to reduce eventual complications.

\section{Bibliography}

1. Gordon AC., et al. "US-guided puncture of the internal jugular vein : complications and anatomic considerations". Journal of Vascular and Interventional Radiology 9 (1998): 333-338.

2. Prabhu MV., et al. "Ultrasound guided femoral dialysis acces placement: a single center randomized trial". Clinical Journal of the American Society of Nephrology 5.2 (2010): 235-239.

3. Massine El Hammoumi., et al. "Incidents and Complications of Permanent Venous Central Acess System". Korean Journal of Thoracic and Cardiovascular Surgery 47 (2014): 117-123.

4. Lefrant JY., et al. "Risk factors of failure and immediate complication of subclavian vein catheterization in critically ill patients". Intensive Care Medicine 28.8 (2002): 1006-1008.

5. Lamkinsi T., et al. "Cathétérisme veineux jugulaire interne : quelle est la meilleure voie d'abord?" Annales françaises d'Anesthésie et de réanimation 31.6 (2012).

6. Xu., et al. "Ultrasound-guided totally implantable venous access ports via the right innominate vein: a new approach for patients with breast cancer". World Journal of Surgical Oncology 17 (2019): 196.

7. Rouzrokh M., et al. "Totally implantable subpectoral vs subcutaneous port systems in children with malignant diseases". Archives of Iranian Medicine 12.4 (2009): 389-394.

8. Samaras P., et al. "Infectious port complications are more frequent in younger patients with hematologic malignancies than in solid tumor patients". Oncology 74 (2008): 237-244.

9. Barbut F., et al. "Cathéters à chambre implantable: épidémiologie des complications et étude microbiologique des dispositifs après ablation". Pathology and Biology 52 (2004): 566-574.

10. Dal Molin A., et al. "The late complications of totally implantable central venous access ports: the results from an Italian multicenter prospective observation study". European Journal of Oncology Nursing 15 (2011): 377-378.

11. Se Jin Ahn., et al. "Ultrasound and Fluoroscopy-Guided Placement of Central Venous Ports via Internal Jugular Vein: Retrospective Analysis of 1254 Port Implantations at a Single Center". Korean Journal of Radiology 13.3 (2012): 314-323.

\section{Assets from publication with us}

- Prompt Acknowledgement after receiving the article

- Thorough Double blinded peer review

- Rapid Publication

- Issue of Publication Certificate

- High visibility of your Published work

Website: www.actascientific.com/

Submit Article: www.actascientific.com/submission.php

Email us: editor@actascientific.com

Contact us: +919182824667 